\title{
Hysteresis Nonlinearities in Pneumatic artificial Muscles
}

\author{
Malak Al-Ma'aita, Moudar Zgoul, Mohammad Al Janaideh
}

\begin{abstract}
Pneumatic artificial Muscles (PAMs) are, nowadays, widely used in factory floor automation as well as in robotics. One of the major appeals about PAMS is the high force to weight ratio as well as their light weight, structural flexibility. Furthermore, PAMS do not exhibit any mechanical wear they are relatively low in cost. However PAMs are known to exhibit hysteresis nonlinearities between the input pressure and the output force as well as the contraction ratio and the input pressure. Such nonlinearities are known to cause oscillations in the open-loop system's responses, as well as poor tracking performance and potential instabilities. This work reviews the some of the available models used to simulate the hysteresis nonlinearities in PAMs which will facilitate to study the effects of these nonlinearities in order to characterize and to enable the design of controllers for compensating for the hysteresis effects.
\end{abstract}

Keywords-PAMs, robotics, modelling, hysteresis, nonlinearities

\section{Introduction}

Recently, PAMs have been widely used in different applications because of their advantages over other types of actuators. In bio-robotic field, for example, the PAMs act as the organic muscle and mimic the humans. Moreover, these systems have been used in biomedical applications. This includes rehabilitation and assisting disabled and paralyzed patients. PAMs are also used in many industrial applications where it can be used in the production lines and industrial tools. However, PAMs exhibit hysteresis nonlinearities between the contraction ratio and the output force as well as the contraction ratio and the input pressure $[1,2]$. These nonlinearities include memory effects, where the output depends not only the current input but also on the past history of the output [3]. Given that PAMs have wide range of applications in many applications and that their performance are strongly limited due to the presence of hysteresis nonlinearities.

These nonlinearities cause oscillations, poor tracking performance, and potential instabilities. Hence, the objective is to overcome these nonlinearities by proposing a nonlinear model in order to enhance the performance in real-time systems.

Moudar Zgoul is with the Mechanical Engineering Department, American University of Madaba Jordan

Malak Al-Ma'aita is with Mechatronics Engineering Department , University of Jordan Amman, Jordan.

Mohammad Aljanaideh is with the deprment of Mechanical and industreial Enginnering, Univesity of Tororno, Ontario, Canada.
A number of reasons cause the hysteresis nonlinearities in the PAMs. The main sources are the compressibility of air, inherent properties of elastic-viscous material, and geometric behaviors of the PAM shell [2]. Hysteresis is referred to the friction between the inner tube and the cords as well as the friction between the cords themselves [1] as illustrated in Figure 1.

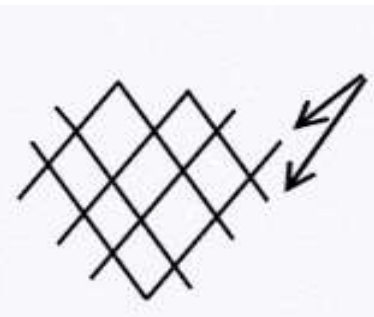

(a)

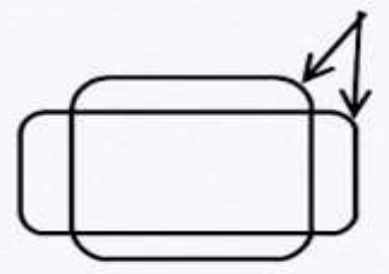

(c)

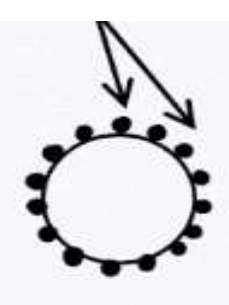

(b)

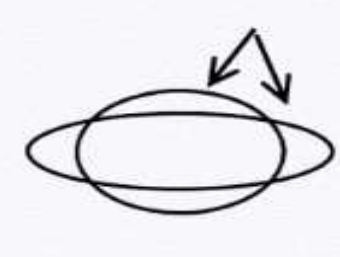

(d)
Figure 1.Causes of Hysteresis in PAM (a) Frictions between cords, (b) bladder and cords friction (c) conical deformation, (d) Bladder stretching in result of decrease in volume

\section{Characterization of Nonlinearities}

\section{A. Experimental work}

Limited numbers of experimental studies were carried out to obtain a deep understanding for how the hysteresis behaves in PAMs. In one of the reported studies [1], two experimental setups were carried out; the isometric setup and the isobaric setup. The aim of the isometric setup, which is also called the constrained model, is to characterize the contracting force. While in the isobaric setup the aim was to measure the hysteresis between the contracting force and the length of the used PAM. Hysteresis in the PAMs appeared in the data obtained by the isobaric setup. In this setup, two fluidic muscles were used; one act as a stretching muscle and the other was the test muscle, where the stretching muscle considered as an actuation for the test muscle. Four bar internal pressure was applied to the test muscle in order to obtain the largest hysteresis loop by considering the full 
stroke loop, which was approximately $40 \mathrm{~mm}$ which is equivalent to $20 \%$ contraction ratio. As concluded in this work, the size and the slope of the hysteresis loop were not affected by varying the excitation frequencies between 0.05 $\mathrm{Hz}$ and $0.8 \mathrm{~Hz}$. In other words, PAMs showed rateindependent behavior in that range of frequencies. The researchers also investigated creep effects, as a result of the temperature rise which leads to bladder relaxation after 50 cycles of 20 seconds for each cycle, resulting in an increase in the contracting force.

In a different study [4] the pressure length hysteresis was obtained differently; isotonic, where in this setup a mass was attached to freely suspended muscle, the mass will go up and down when applying the pressure to the test muscle. The pressure length hysteresis curve exhibits a non-local memory effect as well as a quasi-rate independency characteristic. The quasi-rate-independency was shown at two frequencies of $0.2 \mathrm{~Hz}$ and $0.05 \mathrm{~Hz}$.

Hysteresis between torque and angle in manipulator joint that consisted of two pneumatic muscles of $20 \mathrm{~mm}$ diameter was investigated in a different study [5], two pressure sensors were used to get feedback for the pressure, additionally two load cells to measure the force were used. Pressure inside the muscle was controlled using two proportional valves. The antagonistic setup was obtained by connecting the muscles by means of a timing belt around a rotary joint, where an incremental encoder was used to measure the joint rotation. A data acquisition system was used to collect and process the data points. Initially two experiments were held to check the possibility of using the Maxwell slip model. The purpose of the first experiment is to check the quasi rate independency properties; which can be done by applying a sinusoidal wave on the manipulator arm with two excitation frequencies of 0.192 and $0.044 \mathrm{~Hz}$. The two hysteresis loops obtained for both frequencies matches, therefore as per this work the hysteresis was considered as quasi rate-independent. Initially the two muscles have the same pressure of 3 bar. This value was selected for the pressure since it represents the mean value of pressure, which is calculated from the pressure range that the muscle can withstand ( 0 to 6 bar). The mean pressure yields to a contraction ratio of $12.5 \%$ of their nominal length where the nominal length is $175 \mathrm{~mm}$. The movement of the manipulator was achieved by applying a different pressure to the muscles. In this setup, the hysteresis loop was obtained indirectly using constraint experimental setup where the arm's movement was not allowed.

The dynamic behavior of a PAM used as a part of hand rehabilitation system was reported [6] with the aim of verifying the proposed dynamic model for the used PAMs. The change in length was measured using linear variable differential transformer (LVDT), and a proportional valve with built in pressure sensor was used. In a different study an isobaric setup was performed to measure the force-todisplacement length hysteresis curve for 2, 3, 4 and 5 bar [7] the pressure-to-length hysteresis loops was measured in their study. Hysteresis in PAMs with the pulling forces that were generated using a hydraulic cylinder was measured using a linear variable differential transformer (LVDT) and a pressure transducer was used to measure the pressure of the inlet air [2]

\section{B. Dynamics Modeling}

An accurate dynamic model is essential to attain a control system that can improve the performance of the PAMs. Mathematical model that describe the dynamics of the PAMs can be classified into hysteretic models and nonhysteretic models.

Hysteretic models considered the hysteresis nonlinearities in the dynamics of the PAMs, while the nonhysteretic models ignored the hysteresis nonlinearities. In an attempt to summarize PAMs models a survey on the modeling strategies adopted during last decade is reported [8]. The relationships between pressure, length and force were classified into four model classes. These models included geometrical models, phenomenological biometric/biomechanical models, mathematical models of curved muscles, and empirical models. None of these studies did consider the hysteresis nonlinearities in the dynamics.

Non-hysteretic models included the geometrical models, linear phenomenological models, and empirical models. The geometrical models considered the geometry of the PAMs to model the dynamics between the applied input pressure and the output force. These studies included the most popular geometrical models.

A static model using the principle of virtual work was derived for PAMs under the assumption that the muscle shape is cylindrical [9]. As shown in Figure 2, as the muscle contracts its length decreases while its radius increases. The main drawback of this model is the assumption that the muscle holds its cylindrical shape. This is not accurate during contraction since the muscle usually takes a conic shape at both ends.

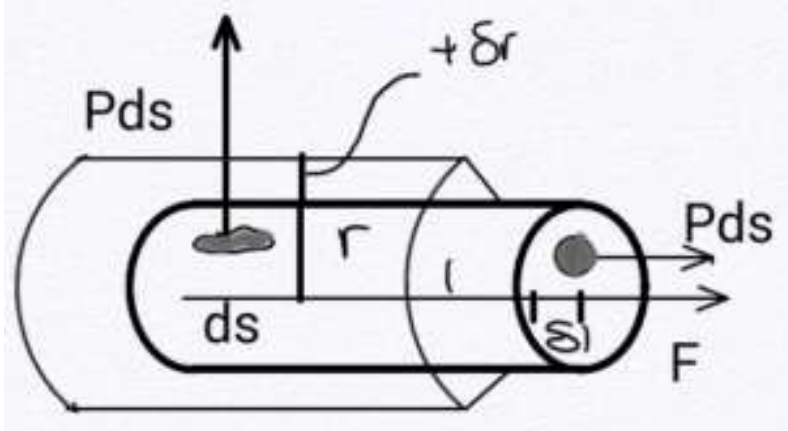

Figure 2 . Virtual work principle applied on Mckebbin muscle

Static physical model for the relation between the tension force and the input pressure for PAMs was proposed in [10] under a number of assumptions. The geometrical models ignored the dynamical behavior of the PAMs since the parameters vary during the actuation process. Furthermore, the models presented in [9 and 10] were developed based on a hypothesis that the wall thickness of the muscle is very low, otherwise the model is not valid as the virtual work principle cannot be applied as the internal pressure does not totally transformed to the braided shell [9]

Characterizing the dynamics of PAMs was considered in physical models constructed with spring-mass system [6, 11]. This model used a contracting element connected in parallel with the spring-mass system as shown in Figure 3. The damping parameter and the stiffness of the spring are 
considered as a polynomial function that depends on the input pressure and the status of the muscle, whether it is in inflation process or the deflation process. However, this model cannot show the hysteresis nonlinearities with memory effects of PAMs.

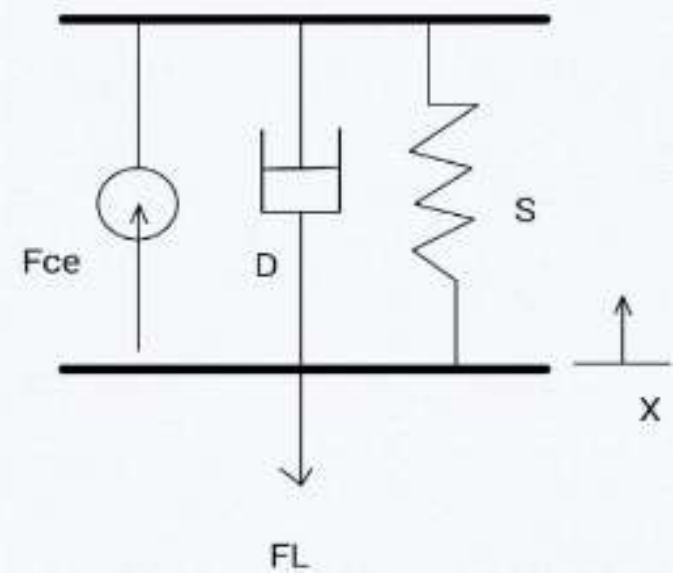

Figure 3. Three element dynamic model of PAM

The empirical model, which is a non-hysteretic model, considered a mechanical spring as shown in Figure 4 to model the dynamics of the PAMs. The nonlinearity between the contraction ratio and the force for different working pressure were modelled considering the PAM as a mechanical spring [2] where a stiffness parameter was assumed as a second order polynomial. This model does not show the hysteresis nonlinearities in the PAMs.

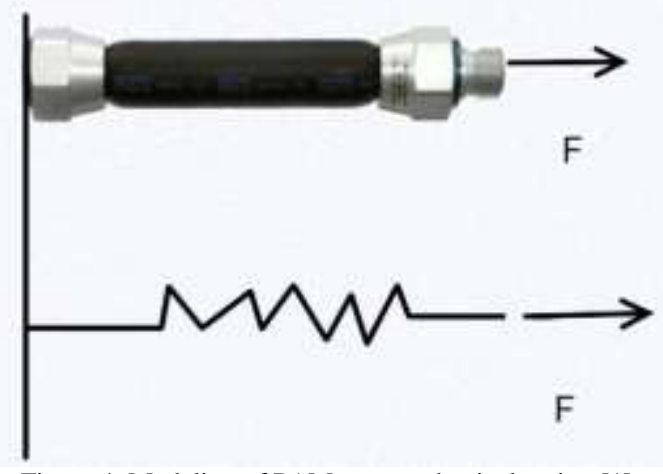

Figure 4. Modeling of PAM as a mechanical spring [1]

The second class of PAMs models is the hysteretic models which models characterize the hysteresis in PAMs. These models include the Maxwell Slip model, which was introduced originally to simulate the behavior of mechanical system under friction [12]. The dry friction behavior is divided into two main regimes: the sliding and the presliding [13]. The Maxwell slip model was constructed based on the analogy between the hysteresis in the muscle and the pre-sliding regime of friction. The Maxwell slip model consists of number of $\mathrm{n}$ elements connected in parallel, each element represented by mass block with coulomb friction and massless linear springs connected together as illustrated in Figure 5.

This model has been employed to quantify force-tolength hysteresis in PAMs [1]. The quasi-rate-independency was shown by applying different frequencies, also the effect of creep due to temperature rise in the bladder was studied.
The main conclusion drawn from this work is that the Maxwell slip model is able to capture the hysteresis nonlinearities at different excitation intervals and internal pressure.

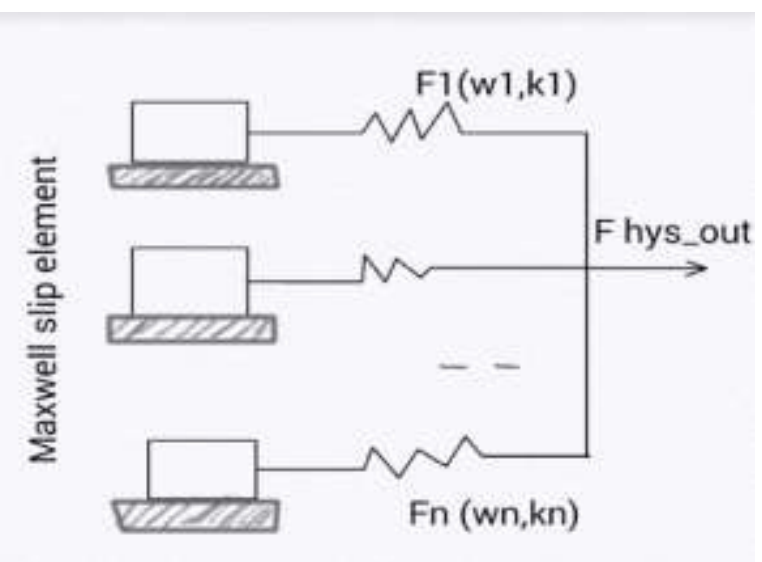

Figure 5. Maxwell slip hysteretic model

The pressure-to-displacement hysteresis nonlinearity for a single PAM was also modeled using Maxwell Slip model [4]. Results showed a quasi-rate-independency characteristic and non-local memory effects. The Hysteresis nonlinearities were not only characterized but also were compensated by developing a control scheme to overcome this nonlinearity where the compensation was carried out by a cascaded controller and the model used in the feedforward path. In the control scheme the purpose of the inner loop and outer loop is to overcome the nonlinearities in the pressure build up and to overcome the nonlinearity in the PAM dynamic, respectively.

Investigation of hysteresis in a manipulator rather than single PAM was reported [5]. Results showed that the hysteresis in the antagonistically system of muscles is similar to the hysteresis characteristics in the single PAM. The modeling of the torque-to-angle hysteresis nonlinearities also was performed with a Maxwell slip model.

The classical Prandtl-Ishlinskii (Pr-Ish) model was used to model the hysteresis between the pressure and the length [7]. Maxwell slip model and Bouc-Wen model were considered. These three models were compared in-term of their ability to capture the hysteresis nonlinearities of the PAMs. Pr-Ish model and Bouc-Wen model showed better results compared to the Maxwell slip model. For Pr-Ish model, the number of play operators was varied between 3 , 9,15 and 20 play operators. The root mean square error between the experimental hysteresis and the simulation output decreases as the number of the play operators increases; with 10.29 to 0.41 RMSE for 3 and 20 play operators, respectively. However, the classic Pr-Ish model has limitation in capturing asymmetric hysteresis as well as rate-dependency [14 and 17]. The generalized Pr-Ish showed better ability to describe material that exhibits symmetric as well as asymmetric minor and major hysteresis loops.

However, a rate dependent Pr-Ish model would have many advantages over other models in terms of controllability. The Pr-Ish model is simple and the inverse can be computed analytically and hence it might prove to be more suitable for real-time applications as in robotics. The main goal of using a rate dependent Pr-Ish model would be 
to characterize and model the hysteresis, and then to facilitate the development of a control approach in order to compensate for the hysteresis nonlinearities and to enhance PAMs performance.

\section{Prandtl Ishliniskii Model}

Prandtl-Ishlinskii model (Pr-Ish) has been widely used to characterize and compensate for hysteresis nonlinearities [15][16]. This model is constructed using play hysteresis operators and quantifies hysteresis nonlinearities by summation of weighted play hysteresis operators. The continuous hysteresis operators which are characterized by the input $v$ and the threshold $r$. Figure 6 illustrates the inputto-output characteristics of the play operator [3].

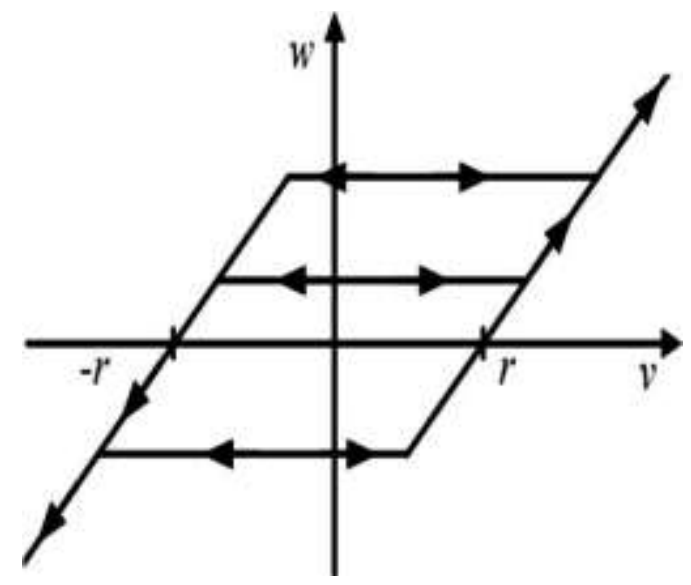

Figure 6. Classical play operator

The Pr-Ish model is expressed as

$$
\Pi[v](t)=q v(t)+\sum_{i=1}^{n} p\left(r_{i}\right) F_{r_{i}}[v](t)
$$

Where the play operator, $F_{r_{i}}[v](t)$ is

$$
\begin{aligned}
& F_{r}[v](t) \\
& =\left\{\begin{array}{c}
\max \left(v(t)-r, w\left(t_{i}\right)\right), \text { for } v(t)>v\left(t_{i}\right) \\
\min \left(v(t)+r, w\left(t_{i}\right)\right), \text { for } v(t)<v\left(t_{i}\right) \\
w\left(t_{i}\right), \quad \text { for } v(t)=v\left(t_{i}\right)
\end{array}\right.
\end{aligned}
$$

Where $r$ refers to the threshold value and $\mathrm{p}$ is the weight function. Figure 7 shows the play operator of the Generalized Pr-Ish model, where this generalized play operator differs from the classical play operator that it yields to zero output at $\zeta$ land $\zeta 2$ which allows describing asymmetric hysteresis loops in addition to the ability of the generalized Pr-Ish to describe the saturation type hysteresis [18]. The value of these constants can be expressed in term of the value of the threshold $\mathrm{r}$ and the envelope functions as:

$$
\begin{gathered}
\gamma_{l}\left(\zeta_{1}\right)=r \text { for } \dot{v}(t)>0 \\
\gamma_{r}\left(\zeta_{2}\right)=-r \text { for } \dot{v}(t)<0
\end{gathered}
$$

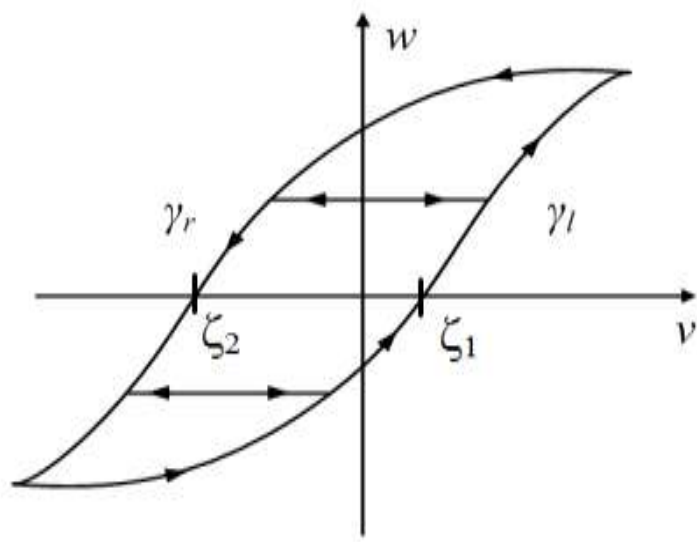

Figure 7. Generalized play operator

The generalized play operator is:

$S_{r}[v(t)]=\left\{\begin{array}{c}\max \left(\gamma_{l} v(t)-r, z\left(t_{i}\right), \text { for } v(t)>v\left(t_{i}\right)\right. \\ \min \left(\gamma_{r} v(t)+r, z\left(t_{i}\right), \text { for } v(t)<v\left(t_{i}\right)\right. \\ z\left(t_{i}\right), \text { for } v(t)=v\left(t_{i}\right)\end{array}\right.$

In the same way the output of the Generalized Pr-Ish model is expressed as the summation of the density function multiplied by the generalized play operator described above.

The rate-dependent Pr-Ish model which is a form of the generalized Pr-Ish model that where the play operators and the threshold values depend as on the rate change of the applied input. The rate-dependent Pr-Ish model is expressed as

$$
\Pi[v](t)=q v(t)+\sum_{i=1}^{n} p\left(r_{i}\right) F_{r_{i(\dot{v}(t))}}[v](t)
$$

Where $\dot{v}(t)$ is the rate is change in input and $F_{r_{i(i(t))}}[v](t)$ is a rate-dependent play operator.

The parameters of the Pr-Ish model can be identified using experimental data. These include the threshold function, play operators, and the density function parameters. After parameter identifications, the hysteresis can be then modeled and thus eliminated. In order to enhance the performance of the PAMs, it is essential to eliminate the hysteresis nonlinearities, which causes inaccuracies and instability in the dynamics of the PAMs. The hysteresis is considered as a problem which affects the performance of the PAMs especially when PAMs used in application where the position must be controlled fast and accurately [11]. Moreover, the hysteresis is an energy loss with amount of the area under the Hysteresis loop [4]. This energy loss leads to complexity in controlling the PAM, and hence it is important to compensate for the hysteresis nonlinearities [1].

\section{Conclusions}

It is essential to model hysteresis nonlinearities in pneumatic artificial muscles (PAMs) as they exhibit rate dependent hysteresis nonlinearities. The Pr-Ish model has many advantages over other hysteresis model such that the 
Pr-Ish is analytical invertible i.e. it has an exact inverse and this can help in hysteresis compensation simply, also the inverse Pr-Ish model is unique, which makes the model attractive for real-time systems. An experimental study in underway to investigate the validity of this model for simulating the hysteresis nonlinearities in pneumatic artificial muscles (PAMs)

\section{Acknowledgments}

The authors would like to thank Abdul Hameed Shoman Foundation for their funding of this research.

\section{References}

[1] Vo-Minh, T. Tjahjowidodo, T. Ramon, H. and Brussel, H.V. "A new approach to modelling hysterises in a pneumatoc artificial muscle using the Maxwell-slip model," IEEE/ASME Transactions on Mechatronics, vol. 16, pp. 177-186, 2011.

[2] Wickramatunge, K.C. and Leephakpreeda, T., "Study on mechanical behaviors of pneumatic artificial muscle," International Journal of Engineering Science, vol. 48, pp. 188-198, 2010.

[3] Al Janaideh, M. Rakheja, S. and Su, C. "An Analytical Generalized Prandtl-Ishlinskii Model Inversion for Hysteresis Compensation in Micro positioning Control," IEEE/ASME TRANSACTIONS ON MECHATRONICS, vol. 16 (4), pp. 734- 744, 2011.

[4] Vo Minh, T. Tjahjowidodo, T. Ramon, H. and Van Brussel, H. "Cascade position control of a single pneumatic artificial musclemass system with hysteresis compensation," Mechatronics, vol. 20, pp. 402-414, 2010.

[5] Vo Minh, T., Kamers, B., Ramon, H. and Brussel, H. "Modeling and control of a pneumatic artificial muscle manipulator joint- Part I: Modeling of a pneumatic artificial muscle manipulator joint with accounting for creep effect," Mechatronics, vol. 22, pp. 923-933, 2012

[6] Xing, K., Haung, J., Wang, Y., Wu, J., Xu, Q. and He, J. "Tracking control of pneumatic artificial muscles actuators based on sliding mode and non-linear disturbance observer," IEEE Control Theory and applications, vol. 4, pp. 2058-2070, 2010.

[7] Chih-Jer Lin, Chii-Ruey Lin, Shen-Kai Yu, and Chun-Ta Chen "Hysteresis modeling and tracking control for a dual pneumatic artificial muscle system using Prandtl-Ishlinskii model," Mechatronics, vol. 28, pp. 35-45, 2015

[8] Kelasidi, E., Andrikopoulos, G. Nikolakopoulos, G. and Manesis, S. "A Survey on pneumatic artificial muscle actuators modeling," Proceedings of the IEEE International Symposium on Industrial Electronics (ISIE), Gdansk, pp. 1263-1269, 27-30 June 2011.

[9] Toundu, B. and Lopez, P. "Modeling and Control of Mckibben Artificial Muscle Robot Actuator," IEEE Control Systems, vol. 20(2), pp. 15-38, 2000.

[10] Chou, C. P. and Hannaford, B. "Measurement and modeling of McKibben pneumatic artificial muscles," IEEE Transactions on Robotics and Automation, vol. 12(1), pp. 90-102, 1996.

[11] Andrikopoulos, G. Nikolakopoulos, G., Arvanitakis, I. and Manesis, S. "Piecewise Affine Modeling and constrained optimal control for a pneumatic artificial muscle," IEEE Transaction of Industrial electronics, vol. 6 (2), pp. 904-916, 2014.

[12] Hassani, V., Tjahjowidodo, T. and Nho Do, T. "A survey on hysteresis modeling, identification and control," Mechanical systems and signal processing, vol. 49, pp. 209-233, 2014.

[13] Parlitz, U. Hornstein, A. Engster, D. Al-Bender, F. Lampaert, V. Tjahjowidodo, T. Fassois, S. Rizos, D. Wong, C. Worden,K. and Manson, G. "Identification of pre-sliding friction dynamics," CHAOS, vol. 14(2), pp. 420-430, 2004.

[14] Al Janaideh, M., Rakheja, S. and Su, C.-Y. "A generalized PrandtlIshlinskii model for characterizing the hysteresis and saturation nonlinearities of smart actuators," Smart Materials and Structures, vol. 18, pp. 1-9, 2009

[15] Al Janaideh, M. and Pavel, K. "Prandtl-Ishlinskii hysteresis models for complex time dependent hysteresis nonlinearities," Physica B, vol. 407, pp. 1365-1367, 2012.
[16] El-shaer, A., Al Janaideh, M, Krejčí, P. and robust performance enhancement using disturbance observers for hysteresis compensation based on generalized prandtl-ishlinskii model. journal of dynamic systems, measurement, and control, 2013, vol. 135, no 5, p. 051008.

[17] Al Janaideh, M., Rakheja, S., Mao, J., \& Su, C. Y. (2009, March). "Inverse generalized asymmetric Prandtl-Ishlinskii model for compensation of hysteresis nonlinearities in smart actuators." In Networking, Sensing and Control, 2009. ICNSC'09. International Conference on (pp. 834-839). IEEE.

[18] Feng, Ying, Camille Alain Rabbath, Henry Hong, Mohammad Al Janaideh, and Chun-Yi Su. "Robust control for shape memory alloy micro-actuators based flap positioning system." In Proceedings of the 2010 American Control Conference, pp. 4181-4186. IEEE, 2010.

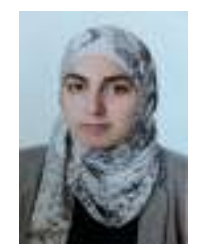

Malak Al Ma'aita received the BSc and M.Sc. degrees in Mechatronics and Mechanical engineering from University of Jordan in 2011 and 2016, respectively. Her research interests are modeling and control of pneumatic artificial muscles.

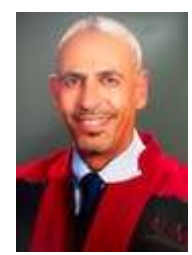

Moudar Zgoul is an Associate Professor of Mechanical Engineering at the American University of Madaba - Jordan. He held various research positions in the UK, Germany and Jordan. He has published research in robotics and in applied mechanics and smart systems.

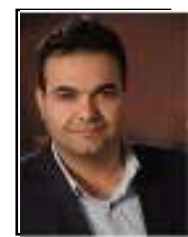

Mohammad Al Janaideh received the MASc. and $\mathrm{Ph} . \mathrm{D}$. degrees in mechanical engineering and mechatronics from Concordia University, Montreal, Quebec, Canada, in 2005 and 2009, respectively. He held research and teaching positions in various countries, including Canada, Jordan, Italy, the USA, and Czech Republic. He joined the Department of Aerospace Engineering, University of Michigan, Ann Arbor, MI, USA, as a Visiting Scholar in 2013. He is with the Department of Mechanical Engineering at University of Toronto. His research interests include control of mechatronics systems, adaptive control for nonlinear systems, modeling and control of pneumatic artificial muscles, and design of MEMS sensors. 